\title{
MUJERES QUE GOBIERNAN MUNICIPIOS EN MÉXICO
}

\author{
DALIA BARRERA BASSOLS \\ Escuela Nacional de Antropología e Historia (México)
}

\section{INTRODUCCIÓN}

Cuando nos abocamos a la reflexión en torno a los avances y retos de la participación política femenina en México, a cinco décadas de la "otorgación» del voto femenino, debemos recordar que si bien es en el año 1947 que se reconoce el derecho al voto de las mujeres mexicanas en el ámbito municipal, no es hasta 1953 que se logra el reconocimiento del derecho de las mujeres a votar y ser votadas en los tres ámbitos políticos: federal, estatal y municipal ${ }^{1}$. Así, vemos aparecer en 1923 (gracias a una legislación local avanzada), a Rosa Torres, la primera regidora municipal, en Mérida, Yucatán; en 1954, a la primera diputada federal, Aurora Jiménez de Palacios, y diez años más tarde, a las dos primeras senadoras: María Lavalle Urbina y Alicia Arellano².

De entonces a ahora, las mexicanas hemos ido ganando espacios de toma de decisiones y de representación popular, como diputadas en los congresos locales, diputadas federales, senadoras, presidentas municipales, síndicas y regidoras, e incluso, dos gobernadoras electas, una interina y una Jefa interina del Gobierno del Distrito Federal. Sin embargo, este avance no ha sido fácil ni el camino suave, existiendo aún serios obstáculos y rezagos, dando lugar a paradojas y contradicciones. La que llama más la atención es aquella que resulta de la cercanía de las mujeres en su condición de madres y esposas, de ciudadanas y de gestoras sociales, con los gobiernos locales (municipales o delegacionales, en el caso del Distrito Federal), y las graves dificultades que han encontrado y encuentran aún ahora para acceder a los espacios de poder y de toma de decisiones en este ámbito de gobierno. Sin embargo, a pesar de la

1. Vid. Barrera, Dalia y Aguirre, Irma: Participación politica de las mujeres. La experiencia de México, México, CONACULTA/INAH, 2003.

2. Agenda Mujeres Año 2000, México, Instituto de la Mujer del Distrito Federal, Gobierno del DF, 2000. 
escasa presencia femenina en las presidencias municipales, resulta interesante preguntarnos acerca de quiénes son las que alcanzan a llegar, qué retos y obstáculos enfrentan, tanto para obtener la candidatura, como durante el proceso electoral, y posteriormente en su ejercicio de gobierno, así como qué es lo que hacen a favor de las mujeres en sus municipios.

\section{REFLEXIONES A CINCO DÉCADAS DEL VOTO FEMENINO EN MÉXICO}

Habiendo transcurrido cerca de cinco décadas desde que se alcanzara el voto femenino en México, tenemos ya cuatro generaciones de mexicanas que han podido ejercer ese derecho ciudadano, durante varias décadas, en el contexto de un régimen autoritario, de partido de Estado de tipo corporativo y clientelar, en el que la gestión de las necesidades de la población se hacía a través de liderazgos de tipo autoritario y caciquil, con fuertes tintes machistas, y donde el respeto al voto representaba más una quimera que un proceso real.

Posteriormente, al producirse el resquebrajamiento de este régimen y el surgimiento de un inacabado sistema de partidos en medio de la lucha por la constitución de un régimen plenamente democrático, encontramos la participación decidida y activa de las mujeres en el proceso de construcción de ciudadanía y de democratización de la vida política y social en nuestro país.

Penoso y difícil ha sido el camino de las mujeres hacia los espacios de poder político y social. A partir de las luchas de las mujeres desde la sociedad civil organizada, desde el movimiento feminista y dentro de los partidos políticos, aunado esto a la presión ejercida por instancias internacionales diversas, que forman parte de la Organización de las Naciones Unidas, así como de organismos financieros como el Banco Mundial y el Banco Interamericano de Desarrollo, los partidos políticos y sus gobiernos han venido abriendo espacios para las mujeres en las altas esferas gubernamentales y de representación popular. De esta manera, tenemos que en 2000, el 15,6\% de los senadores eran mujeres, que el $23,2 \%$ de la cámara de diputados está conformado por mujeres a partir del proceso electoral de 2003, que en 2001 el 14,5\% de los diputados locales eran mujeres y que a partir de 2003 el 33\% de los diputados de la Asamblea Legislativa del Distrito Federal son mujeres también ${ }^{3}$.

Así mismo, encontramos una cierta apertura de la administración pública hacia la presencia femenina, de manera que, para 2000, el 30\% de los funcionarios de la Administración Pública Federal en cargos desde jefatura de departamento hasta secretarías eran del sexo femenino 4 . Paradójicamente, estos datos contrastan fuertemente con aquellos que nos hablan del acceso de las mujeres a espacios de toma de decisiones y a cargos de representación popular en los ámbitos menos espectaculares de gobierno, como serían las presidencias municipales, en donde únicamente el 3,4\% del total estaban ocupadas por mujeres,

3. Datos proporcionados por el Instituto Nacional de las Mujeres, Sistema de Indicadores para el Seguimiento de la Situación de la Mujer, UNIFEM/INEGI/Inmujeres, www.inegi.gob.mx. Datos sobre 2003, en www.cimacnoticias.com/noticias/03jul/030714.html.

4. Barrera, Dalia y Aguirre, Irma: Participación política..., op. cit., p. 37. 
en marzo de $2002^{5}$. El acceso femenino a otros cargos dentro del cabildo ha resultado relativamente menos difícil, de manera que en marzo de 2002 eran mujeres el 6,8\% de los síndicos y el 15,9\% de los regidores ${ }^{6}$.

Carecemos de información suficiente que nos permita hacer un seguimiento de las alcaldesas a lo largo del periodo que va de 1947 a la fecha. Sin embargo, sabemos que, hacia 1986, las alcaldesas eran 69, representando el 2,9\% del total nacional de presidentes municipales; en 1989 fueron $51(2,1 \%)$ y en 1993, $68(2,8 \%)$. De manera que, con ciertos altibajos que reflejan las transformaciones político-electorales en cada región del país, la tendencia a un crecimiento mínimo de la presencia femenina en las presidencias municipales se mantuvo, siendo que para 1985 había 85 presidentas municipales (3,5\% del total del país), en 1998 había 88 (3,6\%) y en 2000, 85 mujeres gobernaban el 3,5\% del total de los municipios.

Tenemos así que de 1986 a 2000, el porcentaje de alcaldesas pasó del 2,9\% al $3, \%$, cifras que nos hablan de un crecimiento muy menor, que se acerca al estancamiento ${ }^{7}$. Nos encontramos así ante la paradoja de que a pesar de que el ámbito local de gobierno constituye aquel espacio en el que transcurre la labor de las mujeres como gestoras de servicios para ellas y sus familias, en su calidad de madres y esposas, así como donde se desarrolla su actividad como pilares de los procesos político-electorales, de defensa del voto y de los derechos ciudadanos, o como importantes bases sociales de diversos movimientos como el campesino, indígena, obrero, etc., resulta que especialmente ese ámbito de gobierno es el que les sigue resultando más inaccesible para lograr formar parte de él y participar e influir en la toma de decisiones.

Este proceso tiene que ver directamente, a mi modo de ver, con lo que ocurre precisamente en los partidos políticos; en 2000 las mujeres constituían más del $50 \%$ de la militancia en los partidos más importantes: el Partido Revolucionario Institucional (PRI), el Partido Acción Nacional (PAN) y el Partido de la Revolución Democrática (PRD), y sin embargo no llegaban a representar más del 33,3\% en el Comité Ejecutivo Nacional, en el mejor de los casos (PRD). Conforme vamos descendiendo hacia los Comités Estatales y más aún, a los Municipales, el acceso de las mujeres va siendo cada vez más difícil, de manera que los espacios locales de toma de decisiones resultan también los de menor acceso femenino, los más cerrados y difíciles, dentro de las propias estructuras partidarias ${ }^{8}$.

5. Barrera, Dalia y Aguirre, Irma: «Liderazgos femeninos y políticas públicas a favor de las mujeres en gobiernos locales en México", en Dalia Barrera y Alejandra Massolo (comps.): El Municipio. Un reto para la igualdad de oportunidades entre hombres y mujeres, México, GIMTRAP,A.C.-InmujeresPNUD, 2003, p. 106.

6. GADSDEn, Carlos: «Democracia, gobiernos municipales y equidad de género en México», en Dalia Barrera y Alejandra Massolo (coords.): Memoria del Primer Encuentro Nacional de Presidentas Municipales, México, Inmujeres, 2003, p. 24.

7. Barrera, Dalia y Aguirre, Irma: «Participación de las mujeres en los gobiernos municipales en México», en Dalia Barrera y Alejandra Massolo (coords.): Memoria del Primer Encuentro..., op. cit., pp. 61-62. 
Es así que en la vida cotidiana y en los espacios de lo local se encuentra un núcleo duro, que concentra la cerrazón, la discriminación y las formas soterradas y pertinaces de disuadir a las mujeres de su incursión hacia el ejercicio pleno de sus derechos ciudadanos. A pesar de todo esto, se van desarrollando en diversos lugares de la República experiencias exitosas de participación femenina en los espacios locales de toma de decisiones. Uno de ellos es el caso del Distrito Federal, en el que se ha vivido un proceso de cambio acelerado en los últimos años, de manera que en el periodo 1997-2000, las mujeres constituyeron el 25,7\% (17) de los Diputados en la Asamblea de Representantes del Distrito Federal, y en 2003, son ya el 33\% (23 diputadas). De otra parte, si en 2000 había seis jefas delegacionales de un total de 16, en 2003 resultan electas cinco, pasando de representar el 37,5\% al 31,2\% del total ${ }^{9}$.

No podemos dejar de lado la evidencia del avance significativo de la presencia femenina debido a la aplicación de las modificaciones en el Código Federal de Instituciones y Procedimientos Electorales, a partir del proceso electoral de 2003 , en el cual los partidos se ven obligados a presentar las candidaturas a diputados y senadores de manera que no se sobrepase el $70 \%$ de ninguno de los géneros. Este aspecto, aunque rebasa los límites de este artículo, debe de llevarnos a la reflexión acerca de las ventajas e inconvenientes de las medidas afirmativas, así como de los límites de éstas, y de las críticas formuladas a los partidos por llevar a cabo prácticas como el ubicar a mujeres en las suplencias, o en las candidaturas en distritos poco probables de ganar, así como los límites de la propia legislación electoral, que exime a los partidos de las cuotas de no sobre-representación, en el caso de votación directa de las candidaturas.

Un ejemplo del avance de las políticas públicas a favor de la equidad de género ocurre a partir de la lucha de diversos grupos feministas y de mujeres preocupadas por la situación femenina desde la sociedad civil y de los partidos, que lleva al nacimiento en 1998 del Instituto de la Mujer del D.F., cuyo trabajo a partir del Sistema de Centros Integrales de Apoyo a la Mujer ha resultado un muy interesante laboratorio en cuanto a la consolidación del proceso de construcción de ciudadanía por parte de las mujeres de las delegaciones, en un mosaico de contextos sociales diversos ${ }^{10}$.

Otro ejemplo lo constituye la gestión de una jefa delegacional con trayectoria feminista, Elena Tapia Fonllem, en la Delegación Iztacalco, cuya experiencia de gobierno se hace acreedora de una mención especial en el II Concurso Regional de Acciones Afirmativas Promotoras de la Participación de las Mujeres en el Poder Local,

8. Barrera, Dalia y Aguirre, Irma: Participación política..., op. cit., p. 46.

9. Ibíd., pp. 43 y 44. Datos para 2003, Consorcio para el Diálogo Parlamentario y la Equidad, en www.cimacnoticias.com/noticias/03 jul/s03070803.html.

10. Pontigo, Josefina: «Reflexiones en torno a una política afirmativa para las mujeres: El Instituto de las Mujeres del Gobierno del Distrito Federal», en Dalia Barrera y Alejandra Massolo (comps.): El Municipio..., op. cit., pp. 55-82. 
realizado en Ecuador en 2003, considerando los esfuerzos de dicha gestión por incidir en la situación y la condición de las mujeres de dicha delegación ${ }^{11}$.

En esta larga historia de participación de las mujeres en todos los rincones de nuestro país, los aportes realizados son valiosos en cada región, en cada municipio. Resulta por ello indispensable crear las condiciones y los espacios para dar a conocer las experiencias, los retos enfrentados y las aportaciones de quienes desde diversas ubicaciones dentro de los gobiernos locales han ido construyendo en la práctica alternativas de atención a las mujeres, que van más allá del enfoque asistencialista, avanzando hacia la construcción de ciudadanía, con base en el cambio cultural que implica la vigencia real de los derechos humanos, políticos y sociales de las mujeres de México. En este espacio de reflexión se pueden visibilizar y poner en la balanza las diversas formas de impulso concreto al cambio social y cultural desde lo cotidiano y micro-social, que sustenta la ciudadanía femenina plena.

El compromiso que adquirió el Estado mexicano al firmar la Convención Sobre la Eliminación de Todas las Formas de Discriminación contra la Mujer (CEDAW) no podrá cumplimentarse si no existen la voluntad y la práctica de formulación de políticas públicas que favorezcan el cambio cultural desde todos los niveles de gobierno, desde el federal y el estatal, hasta el municipal. Mucho tiene que ver esto con el creciente acceso femenino a los espacios de toma de decisiones en los municipios, pero también con las posibilidades reales de que más mujeres y hombres pro-democráticos, sensibles y comprometidos con la igualdad de oportunidades entre los géneros incidan en los espacios de gobierno y en las políticas públicas emanadas de éstos.

Todavía queda entonces un largo camino por recorrer, a favor de la construcción de una sociedad basada en la equidad de género, étnica y social, en México, en la que nos atrevemos a soñar muchos/as mexicanos/as. Pero este proceso debe ser construido desde las bases mismas de la vida social: desde los ciudadanos y las ciudadanas.

Está claro que de poco sirve que se adopte el «punto de vista de género» en los medios oficiales, como resultado de las presiones ejercidas por el Banco Mundial o el Banco Interamericano de Desarrollo, o que se pretenda cumplir "cosméticamente» con los porcentajes de avance hacia la equidad de género del Programa de Naciones Unidas para el Desarrollo, si no se abren los espacios sociales, privados y públicos a una visión democratizadora que abarque los espacios laborales, políticos, gubernamentales y de la vida cotidiana en las colonias, en la familia, en la escuela y en las organizaciones de tipo religioso y cultural. Y, de otra parte, si no existe un compromiso real de los y las políticos/as y funcionarios de ambos géneros, con las necesidades y con la agenda de las mujeres.

En la dirección de este cambio social que vaya de lo público a lo privado y de lo institucional a lo cotidiano, resulta central la participación de las mujeres

11. «Resultados del III Concurso Regional Acciones Afirmativas Promotoras de la Participación de las Mujeres en el Poder Local», en Dalia Barrera y Alejandra Massolo (comps.): El Municipio..., op. cit., pp. 293-300. 
en los gobiernos municipales, como alcaldesas, síndicas, regidoras, funcionarias municipales, así como en su calidad de ciudadanas, colonas, trabajadoras y miembros de los diversos grupos étnicos, con una agenda y propuestas específicas.

\section{MUJERES QUE GOBIERNAN MUNICIPIOS EN MÉXICO. PERFILES Y TRAYECTORIAS}

Como se ha mencionado, el acceso de las mujeres a los cargos en los gobiernos municipales continúa siendo aún muy restringido. Sin embargo, la experiencia de las mujeres que llegan a gobernar un municipio resulta muy valiosa y digna de ser estudiada, desde el punto de vista de los perfiles y trayectorias de quienes síllegan a ese nivel de gobierno, lo que nos dará una idea del tipo de mujeres de que se trata. En este artículo hablaremos de los resultados de una investigación desarrollada en 2002 y 2003, en torno a la presencia femenina en los gobiernos municipales en México ${ }^{12}$. Esta investigación tiene un carácter exploratorio y significa un avance hacia el conocimiento de los perfiles, las trayectorias y las experiencias de gobierno de las mujeres que en México tienen acceso al cargo de presidentas municipales, también llamadas alcaldesas.

Nuestro trabajo se basó en los testimonios y datos aportados por 15 de las 22 alcaldesas asistentes al Primer Encuentro Nacional de Presidentas Municipales, celebrado en junio de 2002, y que formaban parte de las 81 alcaldesas existentes, del total de 2427 presidentes municipales de ambos sexos, en ese momento. Se entrevistó entonces al 18,5\% de las 81 , las cuales representaban a su vez el 3,3\% de los 2427 presidentes municipales.

La exigua presencia femenina en dicho cargo se reflejaba además en el hecho de que, de 31 estados que conforman la República Mexicana, en diez de ellos no existía ni siquiera una mujer gobernando un municipio, en tanto que en 11 de los 22 estados con alcaldesas, únicamente existían una o dos de ellas ${ }^{13}$. Hemos de considerar además que esta condición de marginalidad de las mujeres alcaldesas se expresa también en aspectos como su dispersión a lo largo del territorio nacional, el difícil acceso a ellas, por pertenecer en su mayoría a municipios pequeños o medianos, pobres y muchas veces geográficamente inaccesibles, además de la inexistencia por parte del gobierno federal de un sistema de información básica acerca de los perfiles y datos básicos de los y las presidentes/as municipales. Un ejemplo de estas dificultades lo constituye el hecho de que la información sobre alcaldes, síndicos y regidores en cada municipio no se tiene registrada por sexo, de manera que se trabaja con listados de los cuales se infiere el sexo según el nombre, quedando un cierto margen de error, en el caso de los y las Guadalupe, Refugio, Trinidad, etc.

De cualquier manera, es un hecho claro que ser presidenta municipal en México es aún un caso excepcional, gobernando las mujeres a nivel municipal

12. Barrera, Dalia y Aguirre, Irma: "Participación de las mujeres en los gobiernos municipales ...», op. cit., pp. 57-84.

13. Barrera, Dalia y Aguirre, Irma, "Liderazgos femeninos...», op. cit. 
solamente al 3,4\% de la población nacional, en 2000, y representando tan sólo el 3,3\% del total de presidentes municipales en 2002. Sin embargo, una mujer gobernaba en marzo de ese año en dos de las capitales de estado: Mérida, Yucatán, y Tuxtla Gutiérrez, Chiapas, además de centros urbanos importantes como Gómez Palacio, Durango, Uruapan, Michoacán, Cuautitlán, Estado de México, y Agua Prieta, Sonora. Puede decirse, de cualquier manera, que la mayoría de los municipios gobernados por mujeres se ubican en regiones rurales o semirurales, o son zonas urbanas de tamaño pequeño o medio.

Ser presidenta municipal implica también el haber sido postulada por alguno de los partidos políticos, de acuerdo con lo estipulado en la legislación electoral nacional. Así, en marzo de 2003, el 56,8\% de las alcaldesas en funciones había sido nominada por el PRI, el $17,3 \%$ por el PAN, un $7,4 \%$ por el PRD y el $2,5 \%$ por otro partido. Otro 7,4\% había sido postulada por una coalición de partidos, y el 8,6\% había sido nombrada a través del sistema de usos y costumbres prevalecientes en las comunidades indígenas. De esta manera, el avance en la pluralidad de género en los gobiernos locales de nuestro país parece haberse quedado rezagado con respecto al avance en la pluralidad política ${ }^{14}$. Esto nos habla claramente de graves ausencias en el interior de los partidos políticos, en lo que a promover el acceso femenino a los gobiernos locales se refiere.

A pesar de esta condición de marginalidad de las mujeres en las presidencias municipales, resulta interesante y significativo analizar quiénes son las que llegan a este espacio, cómo lo logran y cómo se desempeñan en dicho cargo, haciendo una comparación con lo que ocurre en otros países de Latinoamérica, de acuerdo con distintos estudios disponibles en la actualidad, abocados a conocer el perfil y las trayectorias de quienes llegan a ese cargo, así como las características de su ejercicio en el poder. Estas comparaciones tienen un carácter aproximativo, teniendo en cuenta que los escasos trabajos existentes son de tipo exploratorio y cualitativo, basándose en entrevistas y cuestionarios a una cierta proporción de las alcaldesas existentes en cada país. Por ejemplo, Jager entrevista a 22 alcaldesas de seis países de Centroamérica, y Arboleda entrevista a 28 en 1991 y a 44 en 1992, para el caso de Ecuador ${ }^{15}$.

Los resultados de la investigación presentados en este trabajo nos han permitido avanzar en ese sentido para el caso mexicano, esbozándose las siguientes conclusiones en cuanto a los perfiles y trayectorias de las alcaldesas.

Su edad, el número de hijos y el estado civil se acercan a los encontrados en los estudios realizados sobre sus homónimas en Costa Rica, El Salvador, Guatemala, Nicaragua, Honduras, Panamá y Ecuador, con la diferencia de que

14. Massolo, Alejandra: «Pluralidad política y pluralidad de género a favor de ayuntamientos democráticos", en Dalia Barrrera y Alejandra Massolo (coords.): Mujeres que gobiernan municipios. Experiencias, aportes y retos, México, PIEM/COLMEX, 1998, pp. 31-48.

15. JAGER, Marcela: Participación politica de las mujeres en los gobiernos locales de Centroamérica, Costa Rica, Fundación DEMUCA-Cooperación Española, 2002 y ARBoleda, María: «Ecuador. Mujeres en el poder local», en IsIS InTERnAcional: El espacio posible. Mujeres en el poder local, Santiago de Chile, Isis Internacional, 1993, pp. 20-43. 
en México no se ubicó a 10 presidentas municipales con edades de la sexta y séptima décadas, como sí se hallaron en los otros países ${ }^{16}$.

De otra parte, si bien en Ecuador se encontró que se trata de mujeres que ya no tienen hijos pequeños, por lo que se hallan menos presionadas por las tareas domésticas y la necesidad de estar presentes en el hogar, el caso de las alcaldesas mexicanas parece acercarse más al de Centroamérica, en donde contaban con el apoyo del servicio doméstico y de parientes cercanos para el cuidado del hogar y de los hijos. Algunas más, a pesar de tener todavía al menos un hijo menor a su cargo, habían pasado la etapa de mayor dependencia física de los hijos. Finalmente, en pocos casos se trataba de mujeres cuyos hijos ya no vivían con ellas, o que carecían de ellos.

Un elemento central en el perfil de las presidentas municipales en México es el de la pertenencia a la capa de población que cuenta con estudios técnicos o profesionales, salvo muy contadas excepciones. En esto coinciden también respecto al perfil educativo de sus pares en otros países latinoamericanos.

Los antecedentes familiares de participación política resultaron significativos en seis de las quince alcaldesas estudiadas, de manera semejante a como se ha visto en Ecuador y a diferencia de lo que se reporta para Centroamérica, en donde la mayoría de las alcaldesas estudiadas tenía esos antecedentes ${ }^{17}$. En el caso de México vemos que las experiencias familiares en la política tenían que ver con la militancia en partidos políticos, como el Partido Revolucionario Institucional, el Partido Acción Nacional y el Partido de la Revolución Democrática.

La participación en clubes sociales, del voluntariado y, en el caso de México, en asociaciones de tipo religioso o profesional, es un factor común entre las alcaldesas de los diversos países latinoamericanos, así como la intervención en la gestión social y comunitaria. Estos antecedentes de participación social diversa están relacionados también con la ubicación social de las alcaldesas, sobre la base de su formación y ejercicio profesional, la cual les da en muchas ocasiones una experiencia de gestión social o comunitaria, de negociación con las autoridades, etc. ${ }^{18}$. Detrás de estas mujeres está entonces una compleja articulación de redes sociales, que se entremezclan con las redes de tipo político, en las que se insertan a través de los partidos políticos y de organizaciones políticas.

Una cuarta parte de las alcaldesas mexicanas estudiadas tenían antecedentes de colaboración en un gobierno municipal y cerca de la mitad del total tenían una militancia en las instancias municipales de un partido político, habiendo recibido además tres alcaldesas una capacitación específica sobre gestión municipal. Cuatro de las presidentas municipales analizadas tenían el antecedente de haber sido candidatas a alcaldesas sin haber sido electas en tres de los casos, pues una de ellas había sido reelecta. De esta manera, estarían lejos de haber

16. Arboleda, María, "Ecuador. Mujeres...», op. cit. y «Mujeres en el poder local», en Jaque al rey. Memorias del Taller Participación política de la mujer, Quito, REPEM, 1994. JAGER, Marcela: Op. cit.

17. Arboleda, María: «Ecuador. Mujeres...», op. cit. Jager, Marcela: Op. cit.

18. Arboleda, María: «Ecuador. Mujeres...», op. cit., pp. 39-40. 
llegado a la presidencia municipal sin ninguna preparación o antecedente sobre la gestión municipal al menos la mitad de los casos de nuestro estudio.

\section{ACCIONES A FAVOR DE LAS MUJERES, OBSTÁCULOS Y RETOS ENFRENTADOS}

En lo que se refiere a las acciones a favor de las mujeres emprendidas dentro de su gobierno, nuestro estudio mostró que la mayoría era sensible a la problemática femenina, aunque casi ninguna había realizado un diagnóstico específico sobre la situación de las mujeres en su municipio. Sin embargo, podemos afirmar, de acuerdo con Arboleda, que las mujeres asumen como "natural» trabajar en pro de las necesidades prácticas de la mujer, por lo que se dirigen en ese sentido hacia las instituciones como el Instituto Nacional del Niño y la Familia, en Ecuador, o el Sistema para el Desarrollo Integral de la Familia (DIF), en México ${ }^{19}$.

Una práctica que encontramos en casi todos los municipios estudiados es la de promover la organización de las mujeres a partir de proyectos productivos, o del DIF, apoyándose en ellos para el mejoramiento de las condiciones de vida de las mujeres. Estos proyectos son altamente valorados por las alcaldesas, nombrándolos en primer lugar al ser interrogadas acerca de su trabajo por mejorar la condición de las mujeres. Se hizo referencia también a la presencia de programas de apoyo a proyectos productivos y al mejoramiento de condiciones de vida, como el PROGRESA, Mujeres en Desarrollo Rural, y otros programas estatales y federales de combate a la pobreza. Únicamente en dos municipios se había creado además una instancia municipal específica para la atención de las problemáticas femeninas.

Sin embargo, más allá de las diversas formas de trabajo con mujeres como los talleres de capacitación, los cursos de manualidades, de costura, de salud reproductiva, los proyectos productivos, la facilitación de recursos para mejorar el nivel de vida, la lucha contra la violencia intra familiar, etc., iniciativas en las que coinciden las alcaldesas de nuestro estudio con las de otros países latinoamericanos ${ }^{20}$, encontramos también incipientes intentos de modificar la situación de subordinación y opresión femenina, con la realización de talleres de autoestima, sobre derechos humanos, etc., así como las referencias de las alcaldesas sobre la necesidad de fomentar los liderazgos y la participación social y política femenina, así como de contribuir a elevar su nivel educativo y profesional. Este punto abre la necesidad de reflexión en torno a la diferencia entre establecer planes, programas y acciones hacia la atención de las necesidades prácticas de las mujeres, y hacerlo contemplando sus necesidades estratégicas. Esta discusión no se ha dado aún de manera colectiva entre las alcaldesas de nuestro país, ni entre las síndicas y regidoras, aunque ya ha comenzado a tener lugar en buena parte de los países latinoamericanos ${ }^{21}$.

19. Ibíd., p. 40.

20. Jager, Marcela: Op. cit., pp. 65-66. ArboledA, María: «Ecuador. Mujeres...», op. cit., pp. 29-33. 
Buena parte de las alcaldesas estudiadas buscaron incluir, desde el primer nivel, en su equipo de trabajo a mujeres como colaboradoras, más allá del propio DIF, en donde todas ubicaron a una mujer como presidenta o como directora, siendo en general profesionales y/o familiares suyas. No faltaron los casos, sin embargo, en los que la presencia femenina en el equipo de gobierno fue muy escasa, ya sea debido al tamaño reducido del municipio, o por encontrarse fuertes problemas para lograr la incorporación de las mujeres como funcionarias municipales o como miembros del cabildo.

En cuanto a los problemas y retos enfrentados, encontramos que, en general, las presidentas municipales de nuestro estudio manifestaron la voluntad de desarrollar una forma de gestión que diera como resultado la transparencia, la honestidad, la eficiencia y la cercanía con la ciudadanía. Enfrentaron sin embargo, en su mayoría, el reto de acceder a una candidatura siendo mujeres, ante las dudas, reticencias y campañas de desprestigio, incluso dentro del partido que las postuló. Al llegar, se encontraron también con la escasez de recursos para el buen desarrollo de su administración, si no fue con verdaderas situaciones de quiebra en las finanzas municipales. Algunas se lanzaron a convencer a la ciudadanía de pagar sus impuestos, otras echaron mano de los recursos familiares para efectuar gastos ineludibles de materiales y equipo para trabajar. Todas se abocaron a "tocar puertas", en los gobiernos estatales o en el federal, en la iniciativa privada, o en asociaciones civiles diversas. Unas cuantas buscaron formar parte de asociaciones formales o informales de municipios de la región, para optimizar recursos, y otras más se abocaron a realizar un "hermanamiento» con alguna ciudad extranjera.

Pero el ejercicio hasta cierto punto marginal de gobernar un municipio por parte de una mujer implicó para las alcaldesas enfrentar no sólo el reto de la escasez de recursos y de superar la lejanía tradicionalmente existente entre el gobierno municipal y la ciudadanía, sino también, en ocasiones, problemas políticos en el cabildo y roces con el gobierno estatal, además de los confictos generados por ser mujer y por ser de oposición. Todo esto ocurre, en general, en medio del predominio de actitudes machistas y de tipo caciquil, en un contexto de violencia hacia las mujeres, en donde las dudas acerca de sus capacidades y de la pertinencia de su presencia en el Palacio Municipal se transforman en campañas de desprestigio en las que se les tacha de ineptas, corruptas, narcotraficantes, etc., y donde proliferan las murmuraciones acerca de su vida personal.

Todo lo expuesto nos confirma la convicción de que los municipios constituyen todavía en nuestro país un duro reto para la equidad de género, tanto como para la convivencia y el gobierno democráticos.

\section{REFLEXIONES FINALES}

El difícil acceso de las mujeres a los cargos en los gobiernos municipales y las diversas problemáticas y retos enfrentados por el hecho mismo de pertenecer al

21. Biasi, Mara D.: «Brasil. El ejecutivo local femenino», en Isis InTERnaCional: Op. cit., pp. 92-93. 
género femenino están relacionados con el contexto sociocultural, que implica el ejercicio de una ciudadanía "peculiar» por parte de las mujeres, sujetas a diversas trabas a su libre circulación, en medio de procesos de violencia familiar y social y de otros mecanismos de control social, así como la confrontación con diversos factores de discriminación en el interior de sus familias, en las comunidades y en las instituciones sociales y políticas.

Se trata de un fenómeno multifactorial, que lleva a que las mujeres de México enfrenten obstáculos a su participación política y social, a su educación formal y a su acceso a espacios de toma de decisiones, tanto en el núcleo familiar y en su socialización primera, como en la escuela, el trabajo y las organizaciones y partidos políticos. Se parte de que el espacio tradicionalmente asignado para ellas es el hogar, por lo que son estigmatizadas, descalificadas y consideradas incapaces o poco confiables, en cuanto ocupan un lugar de liderazgo, ya sea en su barrio, en el sindicato, en el movimiento social, el partido o en el gobierno.

Socializadas como seres-para-otros, sin deseos, necesidades ni proyectos propios, a las mujeres se les hace sentir al poder político y social como algo ajeno, sucio e inaccesible. Sin embargo, paralelamente al avance del proceso de modernización económica y política, a la creciente inclusión femenina en el mercado laboral, a los efectos de la crisis y el empobrecimiento de inmensas capas de la población, a los procesos migratorios que plantean nuevas realidades sociales para hombres y mujeres, etc., va gestándose un acceso creciente a procesos de individuación y empoderamiento personal y colectivo de las mujeres, que se entrecruza con el proceso de construcción de ciudadanía y de búsqueda de formas democráticas de convivencia social y política.

Estas tendencias democratizadoras serán cruciales en el interior de los diversos partidos políticos, para promover la conciencia de la importancia de que exista equidad en el acceso a los gobiernos municipales por parte de hombres y mujeres, para lograr el compromiso de hombres y mujeres de los partidos con una agenda de equidad de género y su inclusión en las plataformas políticoelectorales, así como en la planificación de la vida municipal y de la gestión gubernamental en ese ámbito. De cualquier manera, resulta claro que sin lograr una transformación de la vida cotidiana de los partidos hacia prácticas democráticas y de equidad, será imposible un avance sustantivo en el acceso de las mujeres a los espacios de poder en los gobiernos municipales, como lo muestran algunos estudios sobre el PR ${ }^{22}$.

Ciertamente, el hecho de lograr una significativa mayor presencia de mujeres en los gobiernos municipales no implicará necesariamente mayor sensibilidad de éstas hacia una agenda de las mujeres, pero sí será un factor de equilibrio en la vida social y política en el ámbito municipal, así como un acto de elemental justicia, siendo que, como hemos visto, las mujeres representan cerca del $50 \%$ de la militancia en los tres partidos más importantes. De otra par-

22. Rodríguez Ramírez, Yolanda: "Retos de las mujeres del PRI en las contiendas municipales», en Dalia Barrera (comp.): Participación política de las mujeres y gobiernos locales en México, México, GIMTRAP A.C., 2002, pp. 161-168. 
te, el seguimiento de dicho compromiso sólo será posible si las mujeres de los partidos políticos así como de la sociedad civil ubican la necesidad de impulsar la agenda de las mujeres como prioridad nacional y como aporte femenino a la democratización de la vida social y política, considerando muy necesarios los cambios en el ámbito municipal y en la vida cotidiana.

Finalmente, el avance de la construcción y aplicación de políticas públicas a favor de la equidad de género será una promesa vana sin su concreción cotidiana en los espacios locales. Esto representa un gran reto, al que deben enfrentarse con decisión y claridad las mujeres en los partidos, en los movimientos sociales y en la sociedad civil, en compañía de los hombres sensibles a los problemas de equidad de género.

En este camino, la creación de espacios para el encuentro de las mujeres que participan en los gobiernos locales y para compartir sus experiencias de gestión será crucial en la discusión sobre las estrategias para la construcción de políticas públicas a favor de la equidad de género, más allá de las acciones emprendidas para la atención de las necesidades prácticas de las mujeres, considerando la necesidad de una atención integral de la problemática femenina, que aborde la transformación cultural en todos los aspectos institucionales y de la vida cotidiana.

De otra parte, la «visibilización» de las alcaldesas, síndicas y regidoras a través de la construcción de un sistema adecuado de información sobre su presencia en los diversos municipios del país, sobre sus perfiles y trayectorias, así como la proliferación de estudios que den cuenta de las características de su desempeño en los gobiernos municipales será un aspecto importante para el avance de la organización de las mujeres municipalistas, presidentas municipales, síndicas, regidoras, tesoreras y funcionarias de gobiernos municipales, que constituirá un elemento central en el impulso de las políticas de equidad de género en los municipios, escenario privilegiado de la vida cotidiana de hombres y mujeres en todo el país. 